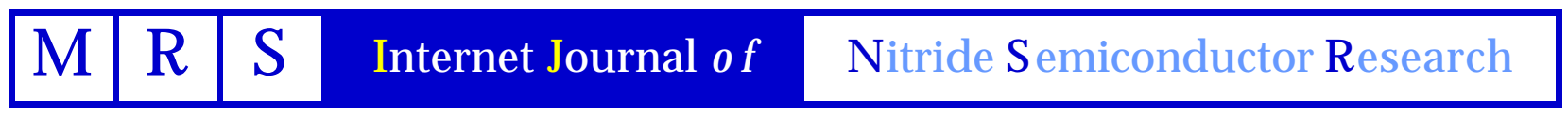

Volume 2, Article 29

\title{
Properties of InGaN deposited on Glass at Low Temperature
}

\author{
Tilman Beierlein, S. Strite \\ IBM Research Division, Zurich Research Laboratory \\ A. Dommann \\ Neu-Technikum Buchs \\ D. J. Smith \\ Center for Solid State Science, Arizona State University \\ This article was received on April 25, 1997 and accepted on September 15, 1997.
}

\begin{abstract}
We have investigated the properties of InGaN grown at low temperature on glass substrates by a plasma enhanced MBE process. The goal of this study was to evaluate the potential of InGaN as an oxide-free, transparent conductor material which could be deposited at or slightly above room temperature with minimal interaction or damage to the underlying material. $\ln _{x} G a_{1-x} N$ films deposited on glass, even without substrate heating, are highly crystalline, but the crystallinity as measured by $\mathrm{x}$-ray degrades at $x<0.5$. The microstructure observed by TEM of InGaN films deposited on unheated substrates is highly columnar, with typical column widths of $\sim 10 \mathrm{~nm}$. The optical absorption spectra of InGaN/glass have a distinct absorption edge at the bandgap, but also high background absorption in the bandgap. $\mathrm{In}_{\mathrm{x}} \mathrm{Ga}_{1-\mathrm{x}} \mathrm{N}$ grown on glass $(\mathrm{x}>0.5)$ is conductive due to its high electron concentration. InN electron Hall mobilities $>20 \mathrm{~cm}^{2} / \mathrm{Vs}$ when grown at $400^{\circ} \mathrm{C}$, and $\sim 7 \mathrm{~cm}^{2} / \mathrm{Vs}$ on unheated substrates were obtained. The addition of $\mathrm{GaN}$ degraded the electrical properties of the films to a greater extent than it improved the transparency. As a result, the best transparent conductor films were pure $\ln N$ which, when deposited at $400^{\circ} \mathrm{C}$, were half as transparent in the green as an indium tin oxide film having the same sheet resistance.
\end{abstract}

\section{Introduction}

\subsection{Purpose of the Investigation}

Transparent conductors are increasingly used in display technology, as static-free coatings, and a host of other applications. Indium tin oxide (ITO) is the most widely used material due to its high transparency throughout the visible spectrum and excellent conductivity when deposited under optimized conditions. However, ITO is not ideal for all transparent conductor applications, for example in oxygen sensitive systems. Furthermore, the elevated deposition and/or post-growth annealing temperatures required to optimize ITO conductivity can be problematic for material systems with poor thermal stability [1] [2]. Alternative transparent conductor materials which are oxygen free, depositable at low temperature, and/or less prone to diffusion into neighboring materials are desirable.

\subsection{Background}

The group III nitrides are attractive candidates for transparent conductor materials and have been investigated for this purpose [3]. Due to their strong bonding and high ionicity, they crystallize easily during deposition and are less prone to trap states. They are therefore likely to retain their conductivity when deposited at low temperature and suffer little outdiffusion of their constituent materials under typical conditions. Finally, the InGaN material system has good transparency in the visible spectrum and is oxygen free. 
The most complete study of GaN for this application was by Sato et al. [3] [4], among others [5], who investigated the properties of GaN deposited onto glass substrates by electron cyclotron resonance (ECR) enhanced MOCVD at various temperatures and gas chemistries. The most conductive GaN/glass was grown at a growth temperature of $350^{\circ} \mathrm{C}$. The conductivity could be further improved by the addition of O [6] or Ti dopants. Optimized GaN/glass had resitivities as low as $\rho=1.8 \times 10^{-2} \Omega \mathrm{cm}$. Typical films had carrier concentrations on the order of $10^{20} \mathrm{~cm}^{-3}$ regardless of the growth conditions. For optimized growth conditions, the resistivity was decreased largely through an increase in the film mobilities. GaN films grown at $150^{\circ} \mathrm{C}$ had resistivities of 2.2 $\Omega \mathrm{cm}$.

\subsection{Present Study}

The goal of the present study was to develop an oxygen-free transparent conductor which could be deposited near room temperature. Since GaN has a bandgap in the near ultraviolet and is transparent throughout the visible spectrum, and InN grown by any technique has been observed to be highly conductive, it was our hope that an optimized $\ln _{x} G_{1-x} N$ alloy would combine the best qualities of both semiconductors and perform well as a transparent conductor. We therefore extended the work of Sato et al. to unheated glass substrates and across the full InGaN alloy system.

\section{Experiment}

\subsection{Substrates}

Common two-inch square square soda-lime glass slides were generally used as substrates. For high temperature $\left(400^{\circ} \mathrm{C}\right)$ depositions, quartz slides were substituted to inhibit impurity outdiffusion from the substrate. The glass was prepared in a clean room with successive washes in acetone and propanol under ultrasound excitation, and rinsed in deionized water. Finally, the glass was blown dry with particle filtered $\mathrm{N}_{2}$, and covered until it was loaded into the vacuum chamber.

\subsection{Growth Chamber}

The deposition chamber shown in Figure 1 is a loadlocked, turbopumped (150 l/s) 8" Conflat (CF) "tee" modified to incorporate a substrate holder/heater. A homemade source flange was mounted on the stem of the tee. It was not possible to rotate the substrate during growth so the effusion cells were focussed on the center of the substrate plane. A thickness and stoichiometry variation of $5-10 \%$ was maintained over the several $\mathrm{cm}^{2}$ used for the measurements described below.

The source flange was originally an 8" CF blank into which three 4.5" water cooled nipples were welded. Two effusion cells (containing In and $\mathrm{Ga}$, and occasionally $\mathrm{Al}$ ) and an ASTeX CECR ${ }^{\mathrm{TM}}$ plasma source (connected to a gas manifold supplying controlled flows of purified and filtered $\mathrm{N}_{2}$ and other gases) were mounted into the source flange nipples. The plasma source had an alumina plasma liner with a $1 \mathrm{~cm}$ aperture. The effusion cells were shuttered to allow flux modulation. The $16 \mathrm{cc}$ effusion cell crucibles were $\sim 20 \mathrm{~cm}$ from the sample surface to limit radiant sample heating, but our chamber was too small to completely eliminate thermal crosstalk. We estimate from thermocouple readings that the unintentionally heated glass substrate temperature during growth was $60-80^{\circ} \mathrm{C}$ depending on the duration of the experiment and the temperature of the Ga cell. For experiments with heated substrates, the temperature was controlled by the thermocouple in contact with the backside of the bonded Varian-style Mo sample holder. It is likely that the actual growth surface temperature was $25-50^{\circ} \mathrm{C}$ higher than the thermocouple reading during these experiments.

\subsection{Characterization}

The InGaN/glass films were characterized by $x$-ray diffraction, optical transmission, Hall characterization, secondary ion mass spectroscopy (SIMS) and transmission electron microscopy (TEM). The film thickness was measured using an Alpha-Step profiler.

\subsubsection{X-ray Diffraction Measurements}

Structural data were recorded with a 4-circle Philips MPD 1880 HRXRD diffractometer equipped with a Ge (220) monochromator (+--+ arrangement) to evaluate the crystallinity of the InGaN/glass thin films. Since the films were polycrystalline, a theta scan sufficed for the structural characterization of the InGaN/glass films. To obtain 
high signal to noise ratios on the thin polycrystalline films, data was often collected 8 hours or more.

\subsubsection{Optical Transmission Measurements}

Optical transmission measurements were performed using an HP 8452A uv-visible spectrometer to evaluate the transparency of various InGaN thin films as a function of wavelength. The spectrum of a blank glass slide was subtracted from the measured InGaN/glass spectrum to obtain the absolute transmission spectra of the thin films. A circular area of roughly $7 \mathrm{~mm}$ diameter was interrogated per sample.

\subsubsection{Hall Measurements}

Hall measurements were performed at room temperature using a $1 \mathrm{kG}$ magnet to evaluate the resistivity, mobility and electron concentration of the InGaN/glass thin films. InGaN/glass samples were cut to the proper size and four In contacts were cold pressed onto the film surface. Our Hall apparatus is capable of measuring mobilities above $\sim 1 \mathrm{~cm}^{2} / \mathrm{Vs}$ and resistivities below $\sim 10^{4} \Omega \mathrm{cm}$. A two point probe with a Keithley picoammeter were used to measure the higher resistivity films. Because of the limited area over which we could grow uniformly, it was possible to fabricate only one $1 \mathrm{~cm}^{2}$ Hall sample per growth run.

\subsubsection{SIMS Measurements}

SIMS measurements were performed in a Cameca IMS $4 \mathrm{f}$ instrument on some of the samples. A description of the SIMS technique is available elsewhere [7] [8].

\subsubsection{TEM Measurements}

Electron micrographs were recorded with a JEM-4000EX HREM instrument operated at $400 \mathrm{keV}$. A description of the TEM technique is available elsewhere [9].

\section{Optimization of the Growth Conditions}

First experiments aimed to establish optimized growth conditions for the experimental arrangement. We systematically varied the deposition parameters, including the $\mathrm{N}_{2}$ flow, microwave plasma excitation power, group III flux and growth temperature. We quickly observed that the most critical parameter is the $\mathrm{N}_{2}$ flow through the plasma source.

\subsection{Variation of $\mathrm{N}_{2}$ Flow and Plasma Power}

Figure 2 shows the experimentally observed deposition rate of $\ln N$ versus the $\ln$ effusion cell temperature as a function of the $\mathrm{N}_{2}$ leak valve setting. Table I correlates the Leybold leak valve settings with the pressure measured in the PEMBE chamber. The deposition rate shows the expected exponential dependence on the effusion cell temperature. However, the InN growth rate increases as much as a factor of three at a given In effusion cell temperature when the $\mathrm{N}_{2}$ flow rate is lowered across the full experimental range.

A similar, but more pronounced (by as much as an order of magnitude) effect was observed in the case of GaN growth (Figure 3). The increase in magnitude for the lighter $\mathrm{Ga}$ atom suggests that some species emanating from the plasma have sufficient energy to sputter adatoms from the growth surface before they incorporate into the film, thereby decreasing the observed growth rate. This suspicion was confirmed by the observation of even more extreme sputtering in the case of AIN growth.

Microwave ECR plasmas are known to produce fewer and less energetic ions at lower operating pressures [10]. However, we were surprised by the magnitude of the sputtering effect with the ASTeX CECR ${ }^{\text {TM}}$, because this plasma source has been used with moderate success by many groups for the PEMBE growth of GaN under similar conditions, including the $1 \mathrm{~cm}$ aperture, but with no mention of sputtering. GaN PEMBE is commonly thought to be constrained by plasma damage to the growing film [11], although recent developments with rf sources have yielded progress. The observed behavior of the ASTeX source is consistent with this opinion.

Figure 2 and Figure 3 show that the sputtering of the deposited film by the plasma can be eliminated by operating in the lower $\mathrm{N}_{2}$ flow regime. Perhaps surprisingly, we observed little or no dependence of the sputtering on the microwave excitation power over the range of $25-200 \mathrm{~W}$, as illustrated in Figure 2 and Figure 
3 by the solid triangles which are data points taken at higher plasma excitation power. These all lie roughly on top of their corresponding $25 \mathrm{~W}$ data points.

We conclude that the kinetic energy distribution of the excited species emanating from the plasma source is mainly influenced by the gas flow, and much less by the microwave excitation power. To ensure gentle deposition conditions, all data reported below were obtained from specimens grown in the low pressure regime using $25 \mathrm{~W}$ microwave excitation power.

\subsection{Influence of the growth rate on electrical properties}

Further experiments investigated the influence of the In flux on the $\ln N$ electrical properties. Figure 4 shows the resitivity of $\operatorname{InN}$ as a function of the In effusion cell temperature under various growth conditions. In the gentle low pressure regime (red and black linear fits of Figure 4), the resitivity increases with In flux. On the other hand, the trend is reversed in the high pressure regime (blue linear fit). A possible explanation is that in the latter energetic ions bombard the surface creating traps and defects. At higher In fluxes, the surface is buried more quickly by adatoms which could reduce the total defect/trap density in the material.

Figure 5 plots the mobility of the $\operatorname{InN}$ thin films as a function of the In cell temperature under various growth conditions. In all $\mathrm{N}_{2}$ flow regimes, the InN mobility is clearly improved by reducing the In flux, and this

improvement accounts for the bulk of the reduction in resistivity shown in Figure 4. The background electron concentration is more or less unaffected by the In flux in the low flow regime, but at higher $\mathrm{N}_{2}$ flow, the increasing plasma damage compensates the $\operatorname{InN}$, reducing the free carrier concentration and making the material more resistive. At the highest In fluxes studied $\left(>1100^{\circ} \mathrm{C}\right)$, the supply of reactive $\mathrm{N}$ was no longer adequate and the film became non-stoichiometric.

Based on these observations, all data reported below were obtained on specimens which were grown at a low target deposition rate of $30 \mathrm{~nm} / \mathrm{hr}$. Typical film thicknesses were $80-200 \mathrm{~nm}$.

\section{Properties of Optimized $\ln _{x} \mathrm{Ga}_{1-x} \mathrm{~N}$ Deposited on Glass Substrates}

\subsection{Experimental Matrix}

An experimental matrix of $15 \ln _{x} G_{a_{1-x}} N$ samples was deposited at constant $N_{2}$ pressure, microwave power excitation, combined group III flux, but at varied growth temperatures and $\mathrm{Ga} / \mathrm{In}$ flux ratios. The specific growth conditions were $4.3 \times 10^{-5}$ Torr (leak valve @ 5.2), $25 \mathrm{~W}, 30 \mathrm{~nm} / \mathrm{hr}$, at temperatures of nominally 60,200 and $400^{\circ} \mathrm{C}$ across the full InGaN alloy range. The unheated and $200^{\circ} \mathrm{C}$ samples were deposited on soda lime glass substrates while the $400^{\circ} \mathrm{C}$ series was deposited on quartz glass. SIMS measurements of the films grown at $200^{\circ} \mathrm{C}$ on soda lime glass detect the presence of $\mathrm{Na}$, and probably due to this contamination, measurements on the $200^{\circ} \mathrm{C}$ series correlate poorly with each other and the lower and higher temperature series. We therefore concentrate the analysis and conclusions on the unheated and $400^{\circ} \mathrm{C}$ series. The experimental matrix is summarized in Table II along with the measured values of the alloy composition and the optical bandgap.

\subsection{X-ray Characterization of InGaN/Glass}

Figure 6 shows $x$-ray data from the InGaN series grown without substrate heating. The complete data are summarized in Table II. In all samples the x-ray spectrum was dominated by a single (002) wurtzite peak indicating that the InGaN alloys were not subject to alloy or crystallographic phase separation. The highest crystallinity was observed in samples containing the highest $\operatorname{lnN}$ mole fraction. InGaN alloys containing $\sim 50 \%$ or less $\operatorname{lnN}$ had markedly worse crystallinity in comparison to more $\mathrm{InN}$ rich material, and GaN, even taking into account the lower x-ray cross section of Ga compared to In.

As the inset shows, the (002) x-ray peaks correlate well with their expected values in the InN rich alloys but the peak positions of the GaN rich alloys correspond to much higher In content than expected from the effusion cell fluxes. This general trend is observable in Table II for the InGaN series grown at each of the three temperatures. In the extreme case, GaN on unheated glass has an x-ray peak which corresponds to $28 \% \operatorname{lnN}$ content. SIMS measurements on a similar GaN/glass film failed to detect the presence of significant In, but detected what was estimated [8] to be $\sim 5 \% \mathrm{O}$ and $\sim 1 \% \mathrm{C}$ contamination. Similar background levels of oxygen and carbon were observed in InN films, also by SIMS. At this time we do not understand the nature of the shift in the x-ray peak at high GaN mole fractions, or why $\mathrm{O}$ or $\mathrm{C}$ contamination might selectively effect the GaN lattice constant more than that of InN. 


\subsection{Optical Absorption Spectra of InGaN/Glass}

Figure 7 shows the optical absorption data from the unheated substrate InGaN series. All layers showed a distinct, roughly linear absorption edge when plotted against the absorption coefficient. Below the bandgap, the spectra differ widely, but the midgap background absorption level was quite high in all cases. The level of this background correlates very well with the x-ray data. The $\operatorname{lnN}$ film, which had the clearest $x$-ray spectrum in terms of peak intensity and FWHM, also has the lowest background optical absorption. Meanwhile, the $\ln _{0.25} \mathrm{Ga}$ $0.75 \mathrm{~N}$ layer has both the highest absorption below the bandgap and the weakest $\mathrm{x}$-ray signal by far, even worse than the GaN layer in both respects. From these data we conclude that the sub-bandgap optical absorption arises from the disordered, and perhaps more contaminated, regions of the film, whereas the highly crystalline volume has a more classical semiconductor absorption contribution to the total spectrum.

We estimate the bandgap of our crystalline semiconductor component by extrapolating the linear absorption edge to its intersection with the midgap absorption minimum. This approximation is schematically illustrated in Figure 7 for the GaN sample, and the values obtained are catalogued in Table II.

We found that the bandgaps obtained by this method correlated suprisingly well with the non-anomalous mole fractions measured by $x$-ray, and less well with the values expected from the effusion cell flux calibrations. Figure $8 \mathrm{a}$ and Figure $8 \mathrm{~b}$ illustrate the the correlation between the $\mathrm{x}$-ray determinations of the mole fraction and the optical bandgap measurements for the unheated substrate series and $400^{\circ} \mathrm{C}$ series respectively. Each data point corresponds to a single sample and measurement. In both cases, the correlation between the $x$-ray and optical absorption data is well described by a linear fit (in Figure 8a, the anomalous GaN point is excluded from the linear fit whereas in Figure $8 \mathrm{~b}$ the $\mathrm{GaN}$ is included because the anomolous shift at $400^{\circ} \mathrm{C}$ is much smaller).

Figure $8 \mathrm{~b}$ also has two data points representing films grown on soda lime glass substrates. Each corresponds closely to its counterpart grown on glass, despite the large level of contamination which can be expected in these films. If the linear fit in Figure $8 \mathrm{~b}$ were made using only the four InN containing datapoints, the GaN points would lie well above the line, similar to the unheated substrate case. If we were to use these data to determine the $\operatorname{InN}$ mole fraction in $\operatorname{lnN}$ rich films, we would indeed choose the linear fit to only the four $\operatorname{lnN}$ containing datapoints.

The measured $\mathrm{InN}$ bandgap at all growth temperatures falls consistently within $\mathrm{E}_{\mathrm{G}}=1.70 \pm 0.03 \mathrm{eV}$, somewhat lower than the generally reported bandgap of $1.9 \mathrm{eV}$ in higher quality $\operatorname{InN}$ [12]. The GaN bandgap determinations >from the absorption data varied from $2.95-3.24 \mathrm{eV}$. The large scatter in the GaN bandgap determinations is probably due less to the absorption edge wavelength than the large variation in the background absorption level between samples. It is notable that the measured bandgaps in all cases studied are smaller by $0.2 \mathrm{eV}$ or more compared to what has been reported in the crystalline InGaN alloy system [12]. Since oxygen contamination in either material could be expected to increase the bandgap, the optical data are not consistent with oxygen contamination significantly altering the bandgap of these semiconductors. We believe that our estimation technique underestimates the true optical bandgap of our material because the extrapolation of the absorption edge is only to the midgap background absorption level minimum. The consistency of the data above $50 \% \operatorname{InN}$ concentration, and its excellent correlation with the x-ray diffraction measurements, suggest that these materials can be well understood as group III nitride polycrystalline semiconductor alloys as opposed to group III oxynitride alloys. Why the GaN rich compounds deviate so widely from their expected behavior is unknown at this time.

\subsection{Electrical Properties of InGaN/glass}

Figure 9 plots the resistivity of the three InGaN series as a function of their $\operatorname{InN}$ mole fraction as determined by $x$-ray. The resistivity of the films is weakly dependent on the growth temperature, but increases rapidly with increasing GaN mole fraction.

Figure 10 plots the mobility of these same samples versus the $\mathrm{InN}$ mole fraction. The mobility shows marked improvement as the growth temperature is increased, but a weak dependence on the $\ln N$ mole fraction for In ${ }_{x} \mathrm{Ga}_{1-\mathrm{x}} \mathrm{N}$ having $\mathrm{x}>0.6$. However, at $\mathrm{x}<0.5$, the mobility falls steeply with increasing GaN content.

Figure 11 shows the corresponding dependence of the free carrier concentration on the alloy composition and growth temperature for these samples. All samples are n-type. Little growth temperature dependence is evident. In the high temperature samples, a strong decrease in the carrier concentration occurs with increasing GaN composition across the entire alloy range, whereas the carrier concentration remains roughly constant at room 
temperature until the GaN mole fraction exceeds $50 \%$.

The electrical characteristics, like the x-ray and optical data, have two regimes which divide near $50 \%$ alloy content. In films composed of $>50 \% \operatorname{InN}$, the electrical properties are dominated by a free electron

concentration which increases rapidly with InN mole fraction, while the free electron mobility of $10-20 \mathrm{~cm}^{2} / \mathrm{Vs}$ is weakly dependent of the composition. The x-ray data of Figure 6 show that the crystal quality of the alloys improves rapidly with increasing $\mathrm{InN}$ mole fraction. It is likely that the increase in carrier concentration at higher InN mole fractions results either from shallower native defect levels resulting from the smaller bandgap or from a reduced deep trap density (higher mobility) corresponding to the improved crystallinity. The constant mobility as a function of InN mole fraction, despite the higher carrier density, indicates that the improvements in crystallinity and reduction of alloy scattering effects at least balance the increased electron-electron scattering.

The electrical properties of our GaN films are orders of magnitude worse than those reported by Sato et al. [4] [5] who used plasma enhanced MOVPE to grow GaN/glass. Unlike these authors, we observe that intentional oxygen (achieved by mixing small flows of $\mathrm{O}_{2}$ with the $\mathrm{N}_{2}$ passing through the plasma source) doping of our GaN (and InN) causes the resistivity to increase markedly whereas they claimed oxygen in their GaN reduced the resistivity. We do not understand the discrepancies between our and Sato et al.'s observations. Intentional $O$ doping was not studied intensively by us because of the danger of destroying our effusion cell heater filaments when running them in an oxygen containing atmosphere.

\subsection{TEM of InGaN/Glass}

Figure 12 shows a TEM image of an $\ln _{0.6} \mathrm{Ga}_{0.4} \mathrm{~N}$ film grown on an unheated substrate (although not a member of the above experimental matrix). What is remarkable about the microstructure is the extreme columnar nature of the growth. Essentially, the thin film is composed of numerous, densely packed, vertical columns roughly 10 $\mathrm{nm}$ wide. The crystal orientation of each column is generally different from its neighbor and no preferred crystal orientation is apparent. It appears that the majority of the volume is crystalline, despite the low temperature growth. We attribute the tendency to form crystalline as opposed to amorphous material to the large bond strength and extreme polarity of the III-V nitrides which energetically favor microscopic crystalline structures.

Figure 13 shows a higher magnification TEM image of the InN film deposited on an unheated substrate which shows one such column of diameter $\sim 10 \mathrm{~nm}$. The column is a defective crystallite of wurtzite InN whose order normal to the substrate is maintained for $50 \mathrm{~nm}$ or more.

The microstructure shown in Figure 12 and Figure 13 is highly unfavorable considering our goal of realizing a transparent (lateral) conductor. Although we haven't directly measured the conductivity of these materials, it is probably highly anistropic. The electrons measured by our Hall experiment have to cross a grain boundary every $10 \mathrm{~nm}$ or so, and are therefore subject to more scattering than would be electrons travelling normal to the substrate along the length of the columnar crystals. We have not yet confirmed whether the columnar microstructure is maintained in InGaN/glass films deposited at higher growth temperatures. If the columnar structure is eliminated by the thermal energy and corresponding adatom mobility made available by substrate heating, we would expect a rapid improvement with increasing temperature of the InGaN electrical properties measured laterally. Figure 9 shows, however, that the resistivity of InGaN/glass varies rather weakly over the temperature range investigated so it seems likely that the $400^{\circ} \mathrm{C}$ films are also columnar.

\section{InGaN/Glass as a Transparent, Oxygen Free Conductor}

The goal of these experiments was to investigate the InGaN system as a potential transparent conductor which could serve in place of ITO. Figure 14 depicts the normalized transmission spectra of various InGaN/glass thin films. To generate these spectra, we normalized the transmittance data to conform with the thickness of each material required to realize a sheet resistance of $100 \Omega$ /square. For example, the $\operatorname{lnN}$ film grown at $400^{\circ} \mathrm{C}$ has a measured sheet resistance of only $45 \Omega$ /square. Therefore, the transmission spectrum of the as grown InN film was recalculated for a thinner film of identical material which would have a sheet resistance of $100 \Omega$ /square. The net effect is that the $400^{\circ} \mathrm{C}$ InN curve is shifted upwards on Figure 14 compared to the raw data. All of the other films have sheet resistances $>100 \Omega$ /square, causing their spectra to be shifted downwards on the $y$-axis when normalized.

Figure 14 indicates that the conductivity of In-rich films generally outweighs the transparency of GaN-rich material, the exception being the unheated substrate case where a $100 \Omega /$ square $\ln _{0.82} \mathrm{Ga}_{0.18} \mathrm{~N}$ film is more transparent in the visible spectrum than its $\ln N$ counterpart. At $\ln N$ content below about $60 \%$, the resistivities of the films are so high that an extremely thick film would be required for a sheet resistance of $100 \Omega /$ square. Due 
to the high background absorption of all our material, their normalized transmission spectra would be nearly zero on Figure 14. In fact, it is the high background absorption present in all of our samples which is most responsible for the poor performance of InGaN compared to ITO in Figure 14. Our best layer, an InN/glass film grown at $400^{\circ} \mathrm{C}$, is roughly half as transparent in the green $(\sim 525 \mathrm{~nm})$ as an ITO film having comparable sheet resistance. Disappointingly, none of the InGaN films grown on unheated substrates show promise as transparent conductors.

\section{Conclusion}

We have investigated a wide variety of physical properties of the InGaN material system deposited on glass substrates at various temperatures by a plasma enhanced MBE process. In the process of optimizing our growth process, we discovered that the ASTeX CECR ${ }^{\text {TM }}$ plasma source is capable of producing species having a kinetic energy large enough to sputter the deposited film, and that careful measures such as reduced gas pressure and plasma power are necessary to avoid this effect.

InGaN films deposited on glass, even without substrate heating, are highly crystalline as evidenced by $\mathrm{x}$-ray and TEM. The crystallinity degrades as the GaN content exceeds $50 \%$. An increase in the background absorption below the bandgap correlated with the reduction in the crystallinity, leading us to postulate that the disordered (and possibly contaminated) component of the films is responsible for the background absorption. We hope to reduce our background absorption in the future by switching to an rf plasma source which should introduce less contaminants and crystal damage than the ASTeX.

The microstructure of InGaN films deposited on unheated substrates is highly columnar, composed of densely packed vertical columns of $\sim 10 \mathrm{~nm}$ width. As a result of the good crystallinity and high background electron concentration, InGaN/glass films are quite conductive, having $\mathrm{InN}$ electron mobilities above $20 \mathrm{~cm}^{2} / \mathrm{Vs}$ and resistivities in the mid $10^{-4} \Omega \mathrm{cm}$ range when grown at $400^{\circ} \mathrm{C}$, and $7 \mathrm{~cm}^{2} / \mathrm{Vs}$ and low $10^{-3} \Omega \mathrm{cm}$ range when grown on an unheated substrate.

All of the films, especially the GaN-rich films, had high optical absorption, even below the bandgap. The hoped-for tradeoff between InN and GaN did not materialize since the addition of GaN degraded the electrical properties to a greater extent than it improved the transparency. Therefore we conclude that InGaN/glass deposited at low temperature is not a promising transparent conductor material system in its own right, although better results may yet be realized with an improved deposition arrangement. On the other hand, InN/glass films deposited at $400^{\circ} \mathrm{C}$ were half as transparent in the green as an indium tin oxide film having the same sheet resistance and may hold some promise for oxygen sensitive transparent conductor applications.

\section{Acknowledgments}

SIMS measurements were performed by Dr. A. Kovarsky and Mrs. M. Jagovkina of the Surface Diagnostics Lab of Mekhanobr Analyt Co., St. Petersburg, Russia under the direction of Dr. A. Shchukarev. We wish to thank Dr. Erica Williams of Laser Enterprise for her assistance with developing the negatives and analysis of the TEM images and diffraction patterns made at ASU.

\section{References}

[1] I. Hamberg, C. G. Granqvist, J. Appl. Phys. 60, R123 (1986).

[2] K. L. Chopra, S. Major, D. K. Pandya, Thin Sol. Films 102, 1 (1983).

[3] H. Sato, T. Mlnami, E. Yamada, S. Takata, M. Ishii, J. Vac. Sci. Technol. A 11, 1422-1425 (1993).

[4] Hirotoshi Sato, Tadatsugu Minami, Eiji Yamada, Makoto Ishii, Shinzo Takata , J. Appl. Phys. 75, 1405-1409 (1994).

[5] S. Zhang, D. E. Brodie, Thin Sol. Films 237, 124-128 (1994).

[6] B-C. Chung , M. Gershenzon , J. Appl. Phys. 72, 651-659 (1992).

[7] A. P. Kovarsky, V. S. Strykanov, MRS Internet J. Nitride Semicond. Res. 1, 34 (1996). 
[8]A. P. Kovarsky, unpublished

[9] D. Chandrasekhar, David J. Smith, S. Strite, M. E. Lin, H. Morkoc, J. Cryst. Growth 152, 135-142 (1995).

[10] A. Ohtani, K. S. Stevens, M. Kinniburgh, R. Beresford, J. Cryst. Growth 150, 902-907 (1995).

[11] S. Strite, H. Morkoçin Handbook of Thin Film Process Technology, Volume A2, , (4, 1995) 1-25.

[12] S. Strite, H. Morkoç, J. Vac. Sci. Technol. B 10, 1237-1266 (1992).

\section{Table I}

Correlation of Leak Valve Setting and PEMBE Chamber Pressure.

\begin{tabular}{|l||l|}
\hline Leybold Leak Valve Setting & PEMBE Chamber Pressure \\
\hline \hline 4.9 & $1.4 \times 10^{-5}$ Torr \\
\hline \hline 5.2 & $4.3 \times 10^{-5}$ Torr \\
\hline \hline 5.5 & $1.4 \times 10^{-4}$ Torr \\
\hline 5.8 & $3.8 \times 10^{-4}$ Torr \\
\hline
\end{tabular}

\section{Table II}

Summary of the experimental $\ln _{x} \mathrm{Ga}_{1-\mathrm{x}} \mathrm{N}$ matrix varying alloy composition and growth temperature.

\begin{tabular}{|c|c|c|c|}
\hline $\begin{array}{l}\text { Target Film } \\
\text { Composition }\end{array}$ & Unheated Substrate $\left(\sim 60^{\circ} \mathrm{C}\right)$ & Substrate Heated to $200^{\circ} \mathrm{C}$ & Substrate Heated to $400^{\circ} \mathrm{C}$ \\
\hline $\ln N$ & $\begin{array}{l}\text { X-ray: } x=1 \\
E_{G}=1.68 \mathrm{eV}\end{array}$ & X-ray: $x=1$ & $\begin{array}{l}\text { X-ray: } x=1 \\
E_{G}=1.67 \mathrm{eV}\end{array}$ \\
\hline $\mathrm{In}_{0.75} \mathrm{Ga}_{0.25} \mathrm{~N}$ & $\begin{array}{l}\text { X-ray: } x=0.82 \\
E_{G}=1.91 \mathrm{eV}\end{array}$ & $\begin{array}{l}\text { X-ray: } x=0.85 \\
E_{G}=1.82 \mathrm{eV}\end{array}$ & $\begin{array}{l}\text { X-ray: } x=0.85 \\
E_{G}=1.85 \mathrm{eV}\end{array}$ \\
\hline $\mathrm{In}_{0.5} \mathrm{Ga}_{0.5} \mathrm{~N}$ & $\begin{array}{l}\text { X-ray: } x=0.60 \\
E_{G}=2.03 \mathrm{eV}\end{array}$ & $\begin{array}{l}\text { X-ray: } x=0.59 \\
E_{G}=2.0 \mathrm{eV}\end{array}$ & $\begin{array}{l}\text { X-ray: } x=0.71 \\
E_{G}=1.99 \mathrm{eV}\end{array}$ \\
\hline $\ln _{0.25} \mathrm{Ga}_{0.75} \mathrm{~N}$ & $\begin{array}{l}\text { X-ray: } x=0.35 \\
E_{G}=2.46 \mathrm{eV}\end{array}$ & X-ray: $x=0.48$ & $\begin{array}{l}\text { X-ray: } x=0.48 \\
E_{G}=2.32 \mathrm{eV}\end{array}$ \\
\hline GaN & $\begin{array}{l}\text { X-ray: } x=0.28 \\
E_{G}=3.10 \mathrm{eV}\end{array}$ & $\begin{array}{l}\text { X-ray: } x=0.27 \\
E_{G}=3.24 \mathrm{eV}\end{array}$ & $\begin{array}{l}\text { X-ray: } x=0.10 \\
E_{G}=3.06 \mathrm{eV}\end{array}$ \\
\hline
\end{tabular}




\section{InGaN Deposition Chamber}
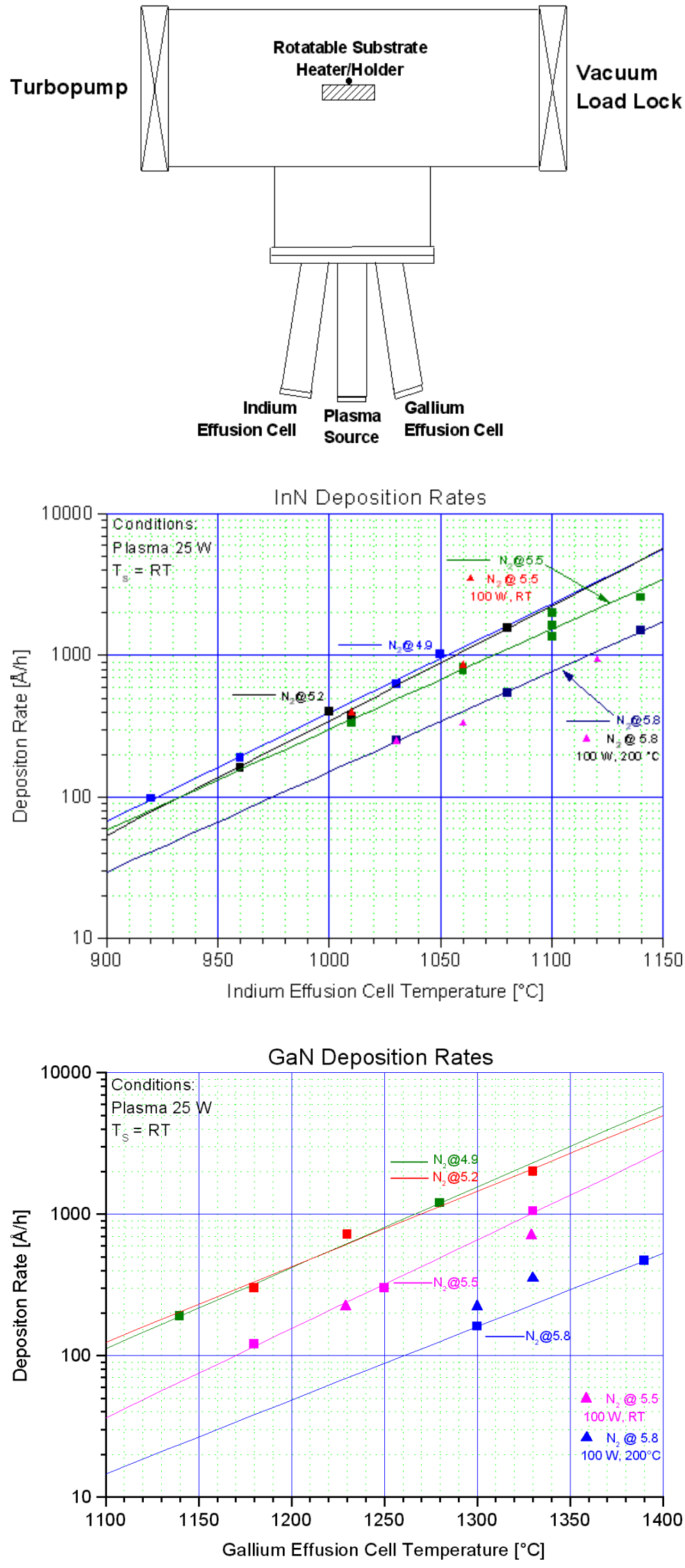

Figure 2. Experimentally observed $\ln N$ deposition rates as a function of $\mathrm{N}_{2}$ flow. By simply varying the $\mathrm{N}_{2}$ flow, the growth rate can be changed by as much as a factor of three.

Figure 3. Experimentally observed GaN deposition rates as a function of $\mathrm{N}_{2}$ flow. The growth rates at the highest flow are an order of magnitude lower than at lower pressures. 

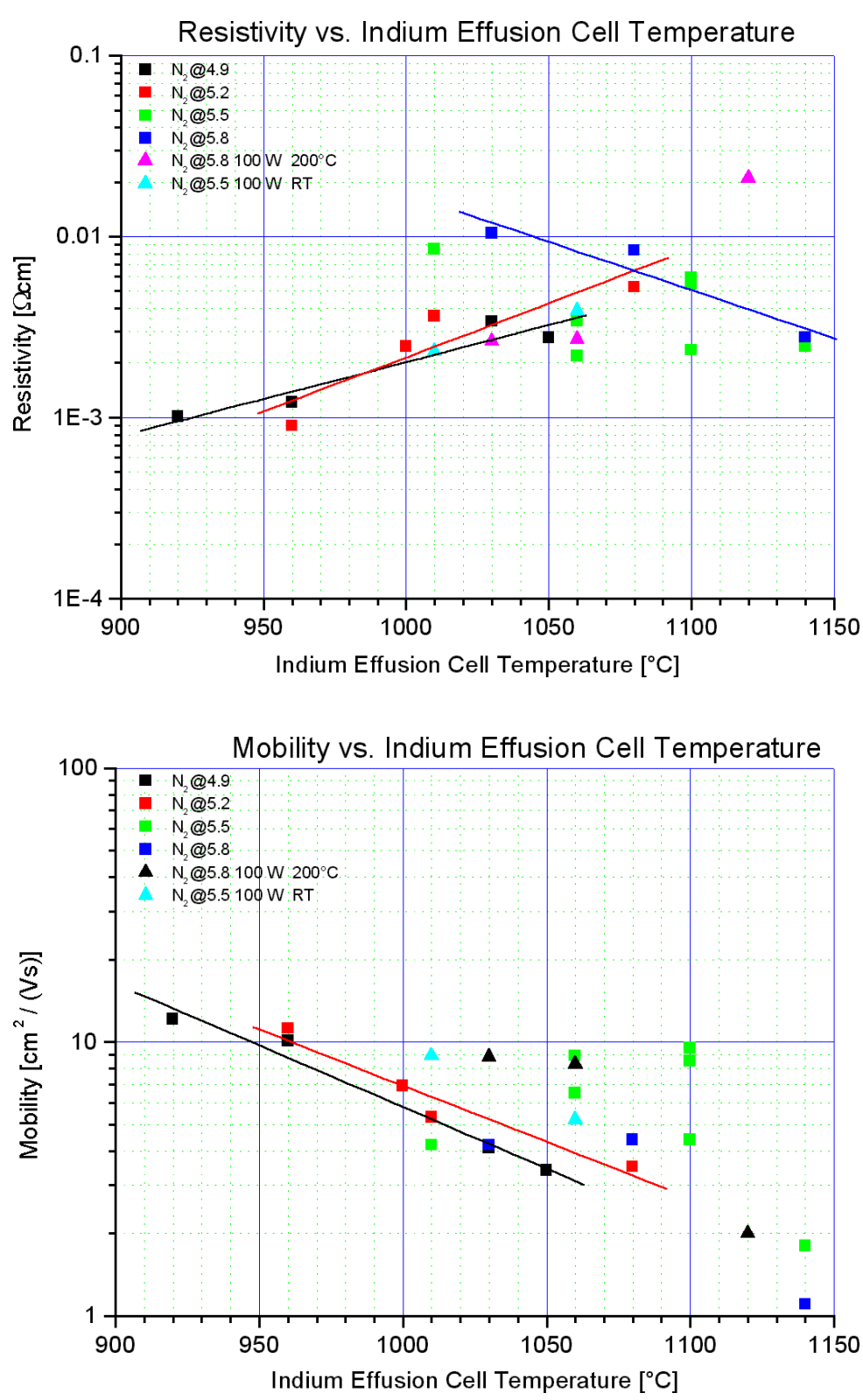

InGaN X-ray Data

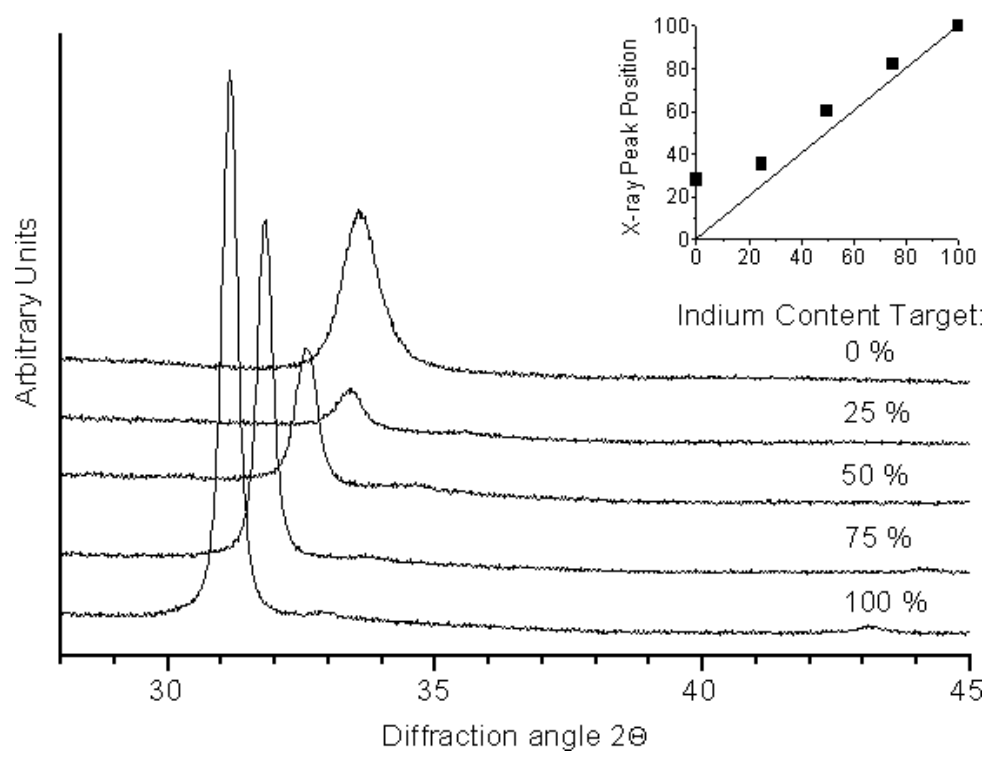

Figure 4. Resitivity of InN vs. In effusion cell temperature under various growth conditions. In the gentle low $\mathrm{N}_{2}$ flow regime (red and black linear fits), the resitivity increases with In flux. In the high $\mathrm{N}_{2}$ flow regime (blue linear fit) the trend is reversed.

Figure 5. Mobility of InN vs. In cell temperature under various growth conditions. In all regimes, the $\operatorname{lnN}$ mobility is generally improved by reducing In flux. The background electron concentration is little affected by In flux in the low $\mathrm{N}_{2}$ pressure regime, but at higher $\mathrm{N}_{2}$ pressures increasing plasma damage compensates the $\operatorname{lnN}$, reducing the free carrier concentration and making the material more resistive.

Figure 6. X-ray data from the unheated substrate InGaN series. Better crystallinity is observed in the films having higher InN mole fraction as evidenced by sharper peaks having higher maximum count rates. The inset plots the In content estimated from the x-ray peak position vs. the value targetted based on the $\mathrm{Ga} / \mathrm{ln}$ flux ratio to highlight the deviation of the wurtzite (002) peak position from the expected value. The deviation grows stronger with GaN content. 

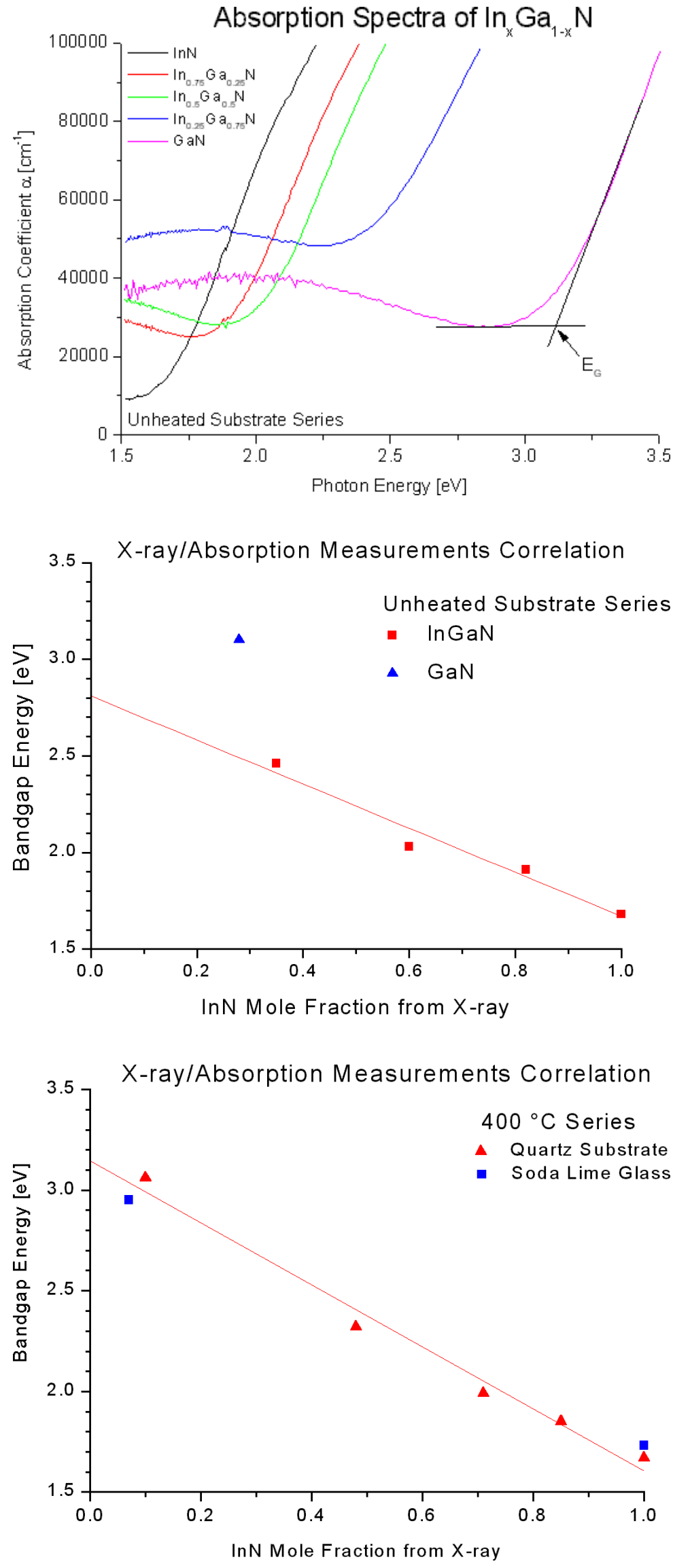

Figure 7. Absorption spectra of the InGaN series grown on unheated glass substrates. The values in the legend are derived from the flux calibrations, and not from direct physical measurements (available in Table II). All of the films show a strong absorption edge which is roughly linear as well as a large background absorption below the bandgap. The bandgaps of these films are estimated after the technique shown schematically for the GaN sample.

Figure 8a. Correlation between the optical bandgap measurement and the $x$-ray peak position for the unheated substrate series. The linear fit was made only to the $\mathrm{InN}$ containing films to illustrate the good correlation between optical and x-ray measurements in these cases, and to highlight the deviation in the GaN film.
Figure 8b. Correlation between the optical bandgap measurements and the $x$-ray peak positions for the $400^{\circ} \mathrm{C}$ series. The linear fit includes the GaN film whose x-ray peak position is only $7 \%$ shifted from the expected value. Had the linear fit been made to only the four $\ln N$ containing data points, the GaN point would lie well above the fit, similar to the unheated substrate case. Also shown are two data points from layers grown on soda lime glass substrates which correlate well with their quartz substrate counterparts. 

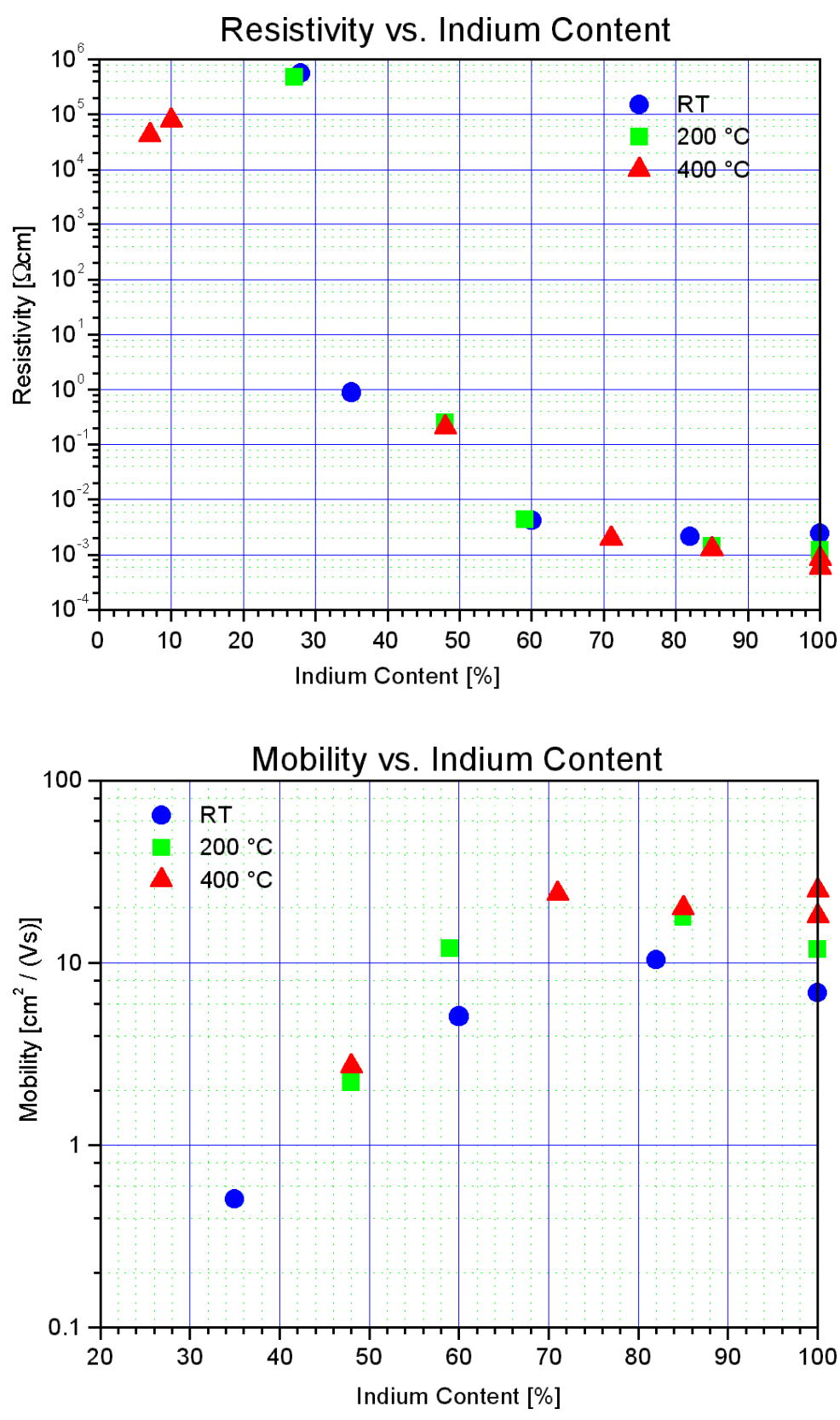

Figure 9. The resistivity of InGaN is weakly dependent on the growth temperature, but increases rapidly with increasing GaN mole fraction. Films grown at lower growth temperatures having less than $25 \% \operatorname{InN}$ mole fraction are too resistive to be measured with our Hall setup so a two point probe is used.

Figure 10. The mobility of $\ln _{x} G a_{1-x} N$ is a strong function of the growth temperature, and decreases with increasing GaN mole fraction at $x<0.5$. Little dependence of the mobility on the $\mathrm{In}_{\mathrm{x}} \mathrm{Ga}_{1-\mathrm{x}} \mathrm{N}$ mole fraction is evident for $\mathrm{x}>0.6$. 


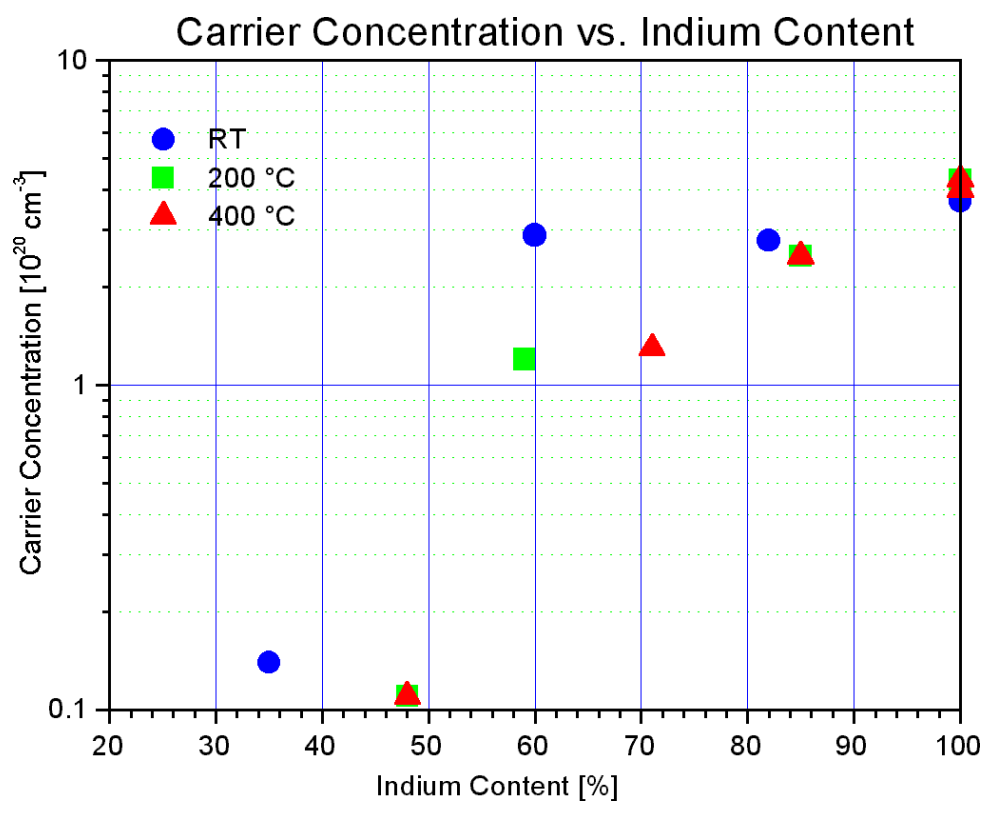

Figure 11. The electron concentration of InGaN is insensitive to the growth temperature. The series grown at higher temperatures demonstrates a monotonic decrease in free carrier concentration as the GaN fraction increases. In the unheated substrate series, the carrier concentration appears to be insensitive to the mole fraction for $\mathrm{InN}$ rich alloys. 


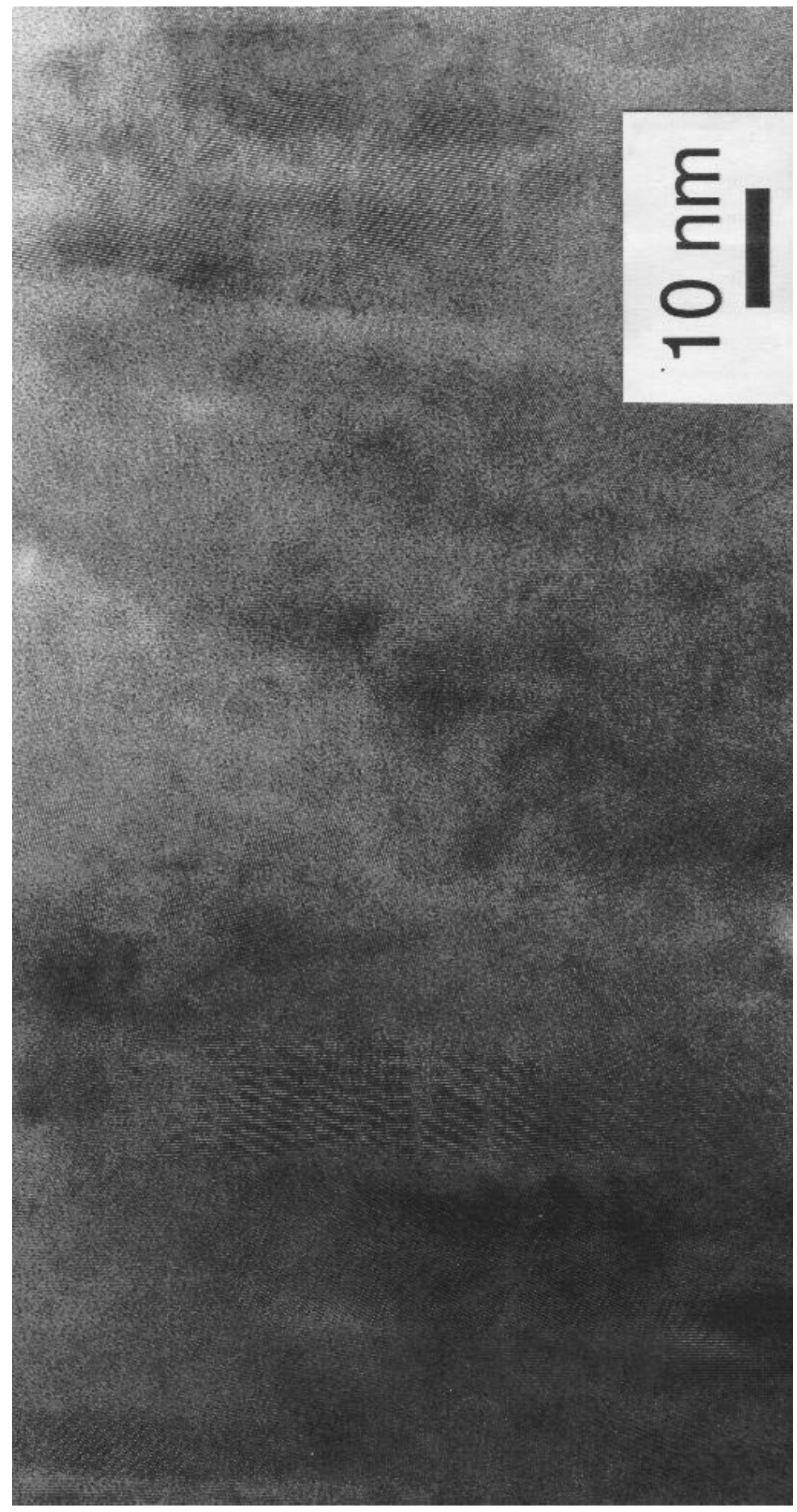

Figure 12. TEM image of an $\ln _{0.6} \mathrm{Ga}_{0.4} \mathrm{~N}$ film grown at room temperature. The microstructure is dominated by densely packed, vertical $10 \mathrm{~nm}$ wide columns. 


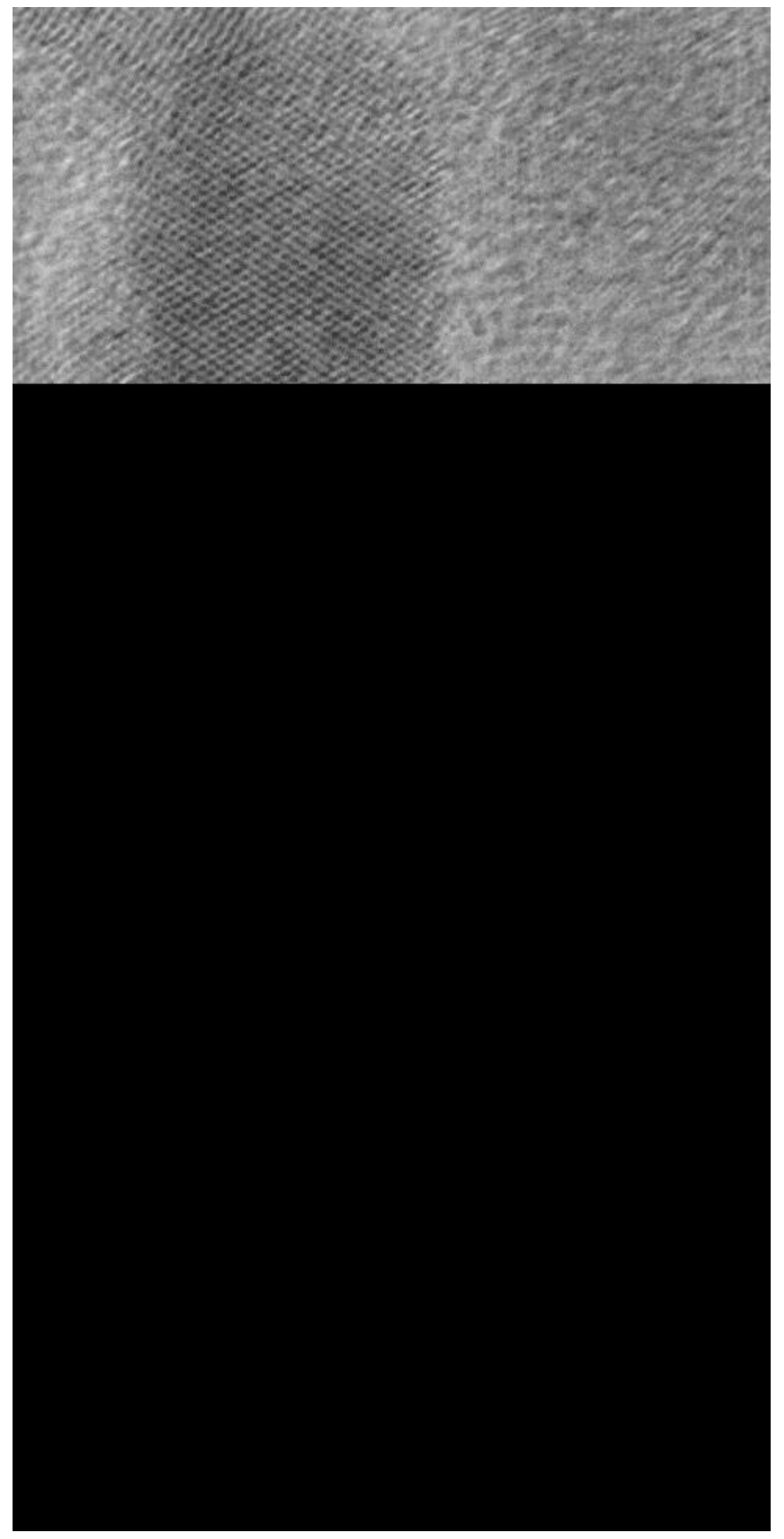

